\title{
Suivi de la contamination humaine en césium à la suite de l'accident de Tchernobyl
}

\author{
Laboratoires d'analyses médicales du groupe CEA*, \\ du service de médecine du travail EdF-GdF**, \\ du service de santé des armées ${ }^{\star \star \star}$
}

(Manuscrit reçu le 7 juillet 1987)

\begin{abstract}
RÉSUMÉ
Les mesures de contrôle effectuées sur le personnel travaillant dans des centres nucléaires français ont permis d'étudier la contamination humaine en césium consécutive à l'accident de Tchernobyl. Environ 800 examens urinaires et 1900 mesures in vivo, effectuées mensuellement, permettent de connaître l'élimination journalière et la quantité fixée dans l'organisme pendant la première année. On en déduit l'ingestion, puis l'exposition. Du fait de la dispersion géographique des centres, la surveillance est représentative d'une grande partie de la France. L'activité des sujets examinés n'a pas encore atteint le maximum après un an. Elle est plus importante à l'est qu'à l'ouest. L'équivalent de dose efficace engagé pendant la première année varie, pour les césium $137+134$, de 6 à $44 \mu$ Sv. Ce bilan, après un an de contrôle, est tout à fait comparable à l'estimation faite à partir des mesures effectuées dans l'environnement les premières semaines après l'accident.
\end{abstract}

\section{ABSTRACT}

The monitoring of workers in french nuclear installations made it possible to study cesium contamination in man following the Chernobyl accident. About 800 urinary bioassays and 1900 in vivo measurements, carried out monthly, showed the daily excretions and body burdens during the first year. Ingestions and exposures were derived. On account of the geographical dispersion of the centres over the country, the monitoring is representative of the larger part of France. Activities in the individuals monitored has not yet reached its maximum values after one year. The committed effective dose equivalents during the first year, for cesium $137+134$, range between 6 and $44 \mu$ Sv. This assessment, after one year's monitoring, is most similar to the evaluation based on environmental measurements made within the first weeks after the accident.

* Participants:

DPS, Fontenay-aux-Roses: L. Jeanmaire, M.L. Daburon. LAM, Cadarache: G. Bataller. LAM, BIII: M.H. Viard, J. Ventadour. LAM, Fontenay-aux-Roses: Ch. Mégemont. LAM, Grenoble: P. Chiadot, M. Millet. LAM, Cogéma La Hague: J.C. Harduin, G. Odilon. LAM, Cogéma Marcoule : J. Mercier, D. Cavadore. LAM, Pierrelatte: J. Chalabreysse, P. Bérard. LAM, Saclay: C. Géronimi, F. Durand. LAM, Valduc: F. Briot.

** SGMT, LAM, St-Denis: C. Chevalier, M. Kwadow.

***CRSSA, Clamart: M. Fatome. 


\section{INTRODUCTION}

L'accident de Tchernobyl a libéré de grandes quantités de radionucléides dans l'environnement et chaque pays essaie de connaître les retombées pour estimer l'exposition consécutive. Des mesures d'activité dans l'air, dans le dépôt, dans la chaîne alimentaire ont été faites dans ce but. La présente étude exploite la surveillance dont fait l'objet le personnel travaillant dans les installations nucléaires de France afin d'estimer la contamination en césium de la population, sa répartition géographique ainsi que son évolution en fonction du temps.

\section{MATÉRIEL, MÉTHODES, RÉSULTATS}

Les résultats proviennent de mesures effectuées au Commissariat à l'énergie atomique (Groupe CEA), au Service de santé des Armées (CRSSA), à Electricité de France (EdF). Ces organismes contrôlent la contamination du personnel par des mesures in vivo et in vitro.

Seuls, les résultats obtenus par les laboratoires d'analyses médicales ont èté exploitées. Ils proviennent de mesures directes par anthroporadiamétrie pour le CEA et les Armées, de mesures dans les urines pour EdF et un laboratoire CEA (tableau I, fig. 1). Chaque mois environ 2700 examens sont pratiqués, dont 1900 in vivo et 800 sur les urines. Les sujets sont des adultes, en majorité de sexe masculin. L'étude de la contamination porte sur un an, de mai 1986 à avril 1987. La répartition géographique de la population surveillée couvre donc une bonne partie de la France. Dans plusieurs régions, les examens ont été effectués par les deux méthodes.

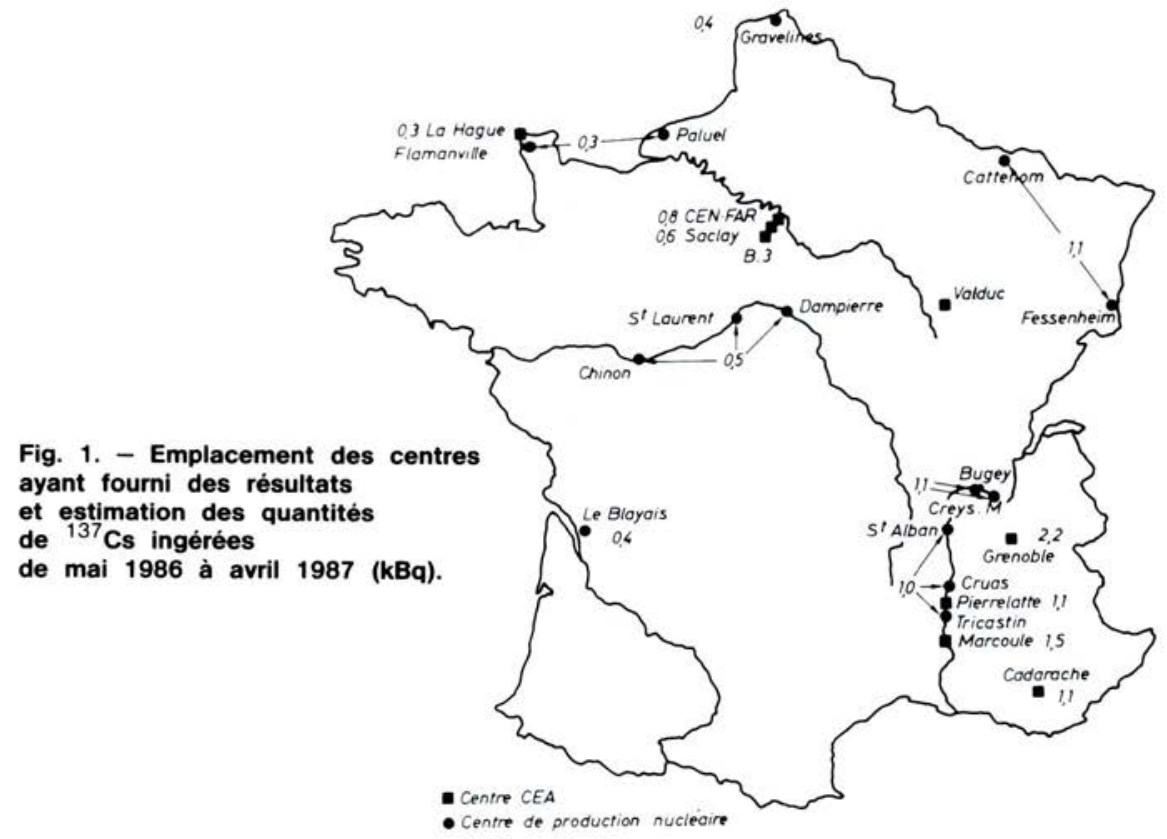


SUIVI DE LA CONTAMINATION HUMAINE EN CESIUM A LA SUITE DE L'ACCIDENT DE TCHERNOBYL

TABLEAU ।

Nombre d'examens mensuels

Mesures in vivo

\begin{tabular}{|l|c|c|c|c|}
\hline & $\begin{array}{c}\text { Nombre } \\
\text { minimal }\end{array}$ & $\begin{array}{c}\text { Nombre } \\
\text { maximal }\end{array}$ & $\begin{array}{c}\text { Nombre } \\
\text { moyen }\end{array}$ & $\begin{array}{c}\text { Nombre } \\
\text { de mois }\end{array}$ \\
\cline { 2 - 5 } Hague & 350 & 509 & 420 & 12 \\
Saclay & 120 & 298 & 220 & 11 \\
Fontenay & 72 & 193 & 125 & 11 \\
CRSSA & & & 13 & 7 \\
BIII & 52 & 178 & 101 & 8 \\
DPS/IPSN & 17 & 19 & 17 & 8 \\
Valduc & & & 75 & 5 \\
Marcoule & 445 & 633 & 578 & 12 \\
Pierrelatte & 51 & 122 & 64 & 12 \\
Grenoble & 58 & 158 & 113 & 12 \\
Cadarache & 64 & 280 & 205 & 11 \\
\hline
\end{tabular}

Mesures sur les urines

\begin{tabular}{|l|c|c|c|c|}
\hline & $\begin{array}{c}\text { Nombre } \\
\text { minimal }\end{array}$ & $\begin{array}{c}\text { Nombre } \\
\text { maximal }\end{array}$ & $\begin{array}{c}\text { Nombre } \\
\text { moyen }\end{array}$ & $\begin{array}{c}\text { Nombre } \\
\text { de mois }\end{array}$ \\
\cline { 2 - 5 } Nord & 33 & 144 & 84 & 12 \\
N.O. & 36 & 128 & 61 & 12 \\
Centre & 49 & 140 & 112 & 12 \\
S.O. & 40 & 75 & 56 & 12 \\
N.E. & 34 & 109 & 53 & 12 \\
Est & 57 & 176 & 100 & 12 \\
S.E. & 42 & 269 & 180 & 12 \\
Cadarache & 98 & 271 & 148 & 12 \\
\hline
\end{tabular}

Nord = Gravelines.

N.O. $=$ Paluel, Flamanville.

Centre $=$ Dampierre, St-Laurent, Chinon.

S.O. = Le Blayais.

N.E. = Fessenheim, Cattenom

(à partir du 11/86).

Est = Bugey, Creys-Malville.

S.E. = Cruas, Tricastin, St-Alban

(à partir du 10/86).

VOL. $22 \cdot \mathrm{N}^{\circ} 4$ 
Les mesures in vivo proviennent de 11 laboratoires. Chacun dispose éventuellement de plusieurs chaînes de spectrométrie gamma munies d'un gros détecteur en $\mathrm{Nal}(\mathrm{TI})$ placé dans une enceinte de protection pour diminuer le mouvement propre. Bien que semblables dans le principe, les installations peuvent différer sur des points importants tels que la nature et la taille de la protection, les caractéristiques du détecteur, le mode d'emploi.

- Une intercomparaison réalisée en 1980 avec des fantômes contenant $3,7 \mathrm{kBq}$ de césium 137 et $3,7 \mathrm{kBq}$ de cobalt 60 a donné des résultats plutôt bien groupés. Pour 6 laboratoires, l'écart était inférieur à $10 \%$, pour 3 inférieur à $20 \%$; une nouvelle installation donnait des résultats surestimés de $60 \%$ (facteur de géométrie sous-estimé). Pour des niveaux 10 à 50 fois plus faibles, et un spectre plus compliqué que celui du césium 137 seul, des divergences plus accentuées sont possibles. Les calculs pour obtenir le césium 137 et, éventuellement, le césium 134 peuvent différer d'un laboratoire à l'autre. Par contre, chaque laboratoire travaillant de la même façon au cours du temps, et le nombre d'examens pratiqués par mois dépassant souvent la centaine, la moyenne représente, sans doute, fidèlement l'évolution de la contamination en un lieu donné.

- La limite de détection estimée pour un individu standard varie de 40 à $160 \mathrm{~Bq}$ selon les installations et les conditions d'emploi. En pratique, chaque laboratoire indique mensuellement le nombre d'examens pratiqués, le nombre de résultats non significatifs, la valeur la plus faible, la valeur la plus forte, la moyenne et l'écart type. Les valeurs au-dessous du seuil de détection sont affectées de la valeur 0 . Le tableau II indique les valeurs moyennes mensuelles de l'activité et le tableau III les pourcentages de résultats positifs; cette dernière information permet de mieux apprécier la confiance que l'on peut accorder à la moyenne. Les personnes extérieures à la région et les cas de contamination professionnelle ne sont pas pris en compte dans cette enquête.

Les urines proviennent de 14 centres de production nucléaire (CPN) d'EdF et du centre d'études nucléaires de Cadarache. Certains CPN étant assez proches, les résultats ont été réunis par zone de façon à disposer d'un nombre suffisant de données. Les mesures des 7 régions ainsi obtenues sont effectuées dans un seul laboratoire au moyen de détecteurs au germanium permettant de détecter dans les meilleures conditions $0,3 \mathrm{~Bq} / \mathrm{l}$ en césium 137. La comparaison des résultats par région n'est donc pas soumise à des variations interlaboratoires. Les résultats (tableaux II et III) sont présentés de la même façon que ceux des mesures in vivo.

Les activités ingérées sont calculées en utilisant la formule de rétention de la CIPR 30 [1]:

$$
r(t)=0,1 e^{-\frac{0,693}{2} t}+0,9 e^{-\frac{0,693}{110} t}
$$

oủ 2 et 110 représentent les périodes biologiques exprimées en jours, et la fraction du débit d'origine systémique transférée dans les urines [4]:

$$
f_{u}=0,80
$$


SUIVI DE LA CONTAMINATION HUMAINE EN CESIUM A LA SUITE DE L'ACCIDENT DE TCHERNOBYL

II est admis que, chaque mois, la contamination journalière reste constante. A partir des résultats des mesures in vivo et urinaires, il a été possible de calculer l'activité totale ingérée en un an (tableau IV) et l'activité journalière moyenne ingérée durant chaque mois (tableau V).

\section{DISCUSSION}

Plusieurs remarques importantes doivent être faites:

- avant l'accident, le seul radionucléide assez aisément détectable était le potassium 40 naturel. Depuis, les césium 137 et 134 ont été mis en évidence plus ou moins facilement selon le degré de contamination;

- dans le cadre de la surveillance systématique, les cas de contamination professionnelle sont plutôt rares: il s'agit alors d'un ou plusieurs radionucléides caractéristiques du lieu de travail;

- les activités retrouvées sont au moins trois ordres de grandeur en-dessous de la limite d'incorporation annuelle pour les travailleurs et, de ce fait, elles ne sont pas prises en compte pour établir un bilan d'irradiation professionnelle. II n'était donc pas du tout certain que l'utilisation secondaire d'examens réalisés dans un but professionnel permette des comparaisons sur la population, car les activités se situant bien en-dessous des valeurs significatives en radioprotection, les méthodes de mesure pouvaient être inadaptées.

\section{Activités mesurées (tableaux II et III)}

Pour les mesures in vivo, dès le mois de juillet, les pourcentages de résultats positifs atteignent ou dépassent $90 \%$, sauf à La Hague. Pour les urines, ces pourcentages n'atteignent jamais $100 \%$. Les moyennes sont calculées en adoptant la valeur 0 pour les résultats non significatifs, elles sont ainsi sous-estimées. La sous-estimation est surtout importante pour les premiers mois où les activités moyennes sont faibles, voire nulles. D'autre part, le regroupement par zone a l'inconvénient de masquer des variations locales. Le cas le plus net est celui des CPN de Cruas et du Tricastin. Les activités moyennes à Cruas sont supérieures à celles du Tricastin d'environ $50 \%$ jusqu'à fin octobre et d'environ $20 \%$ depuis.

Les figures 2 et 3 illustrent l'évolution de l'activité moyenne en quelques points caractéristiques choisis dans le tableau II. II a été demandé aux participants de calculer l'écart type. En ce qui concerne les mesures in vivo, le coefficient de variation est généralement de 30-40\%; on trouve quelques valeurs au-delà de 3 écarts types dans tous les centres. Pour les urines, le coefficient est plus important, de l'ordre de $100 \%$; ceci peut être dû à la mesure (activités très faibles, utilisation de plusieurs détecteurs d'efficacité différente); en outre, l'élimination urinaire est plus soumise aux variations journalières du césium dans l'alimentation que la charge corporelle mesurée in vivo.

VOL. $22 \cdot \mathrm{N}^{\circ} 4$ 
SUIVI DE LA CONTAMINATION HUMAINE EN CÉSIUM A LA SUITE DE L'ACCIDENT DE TCHERNOBYL

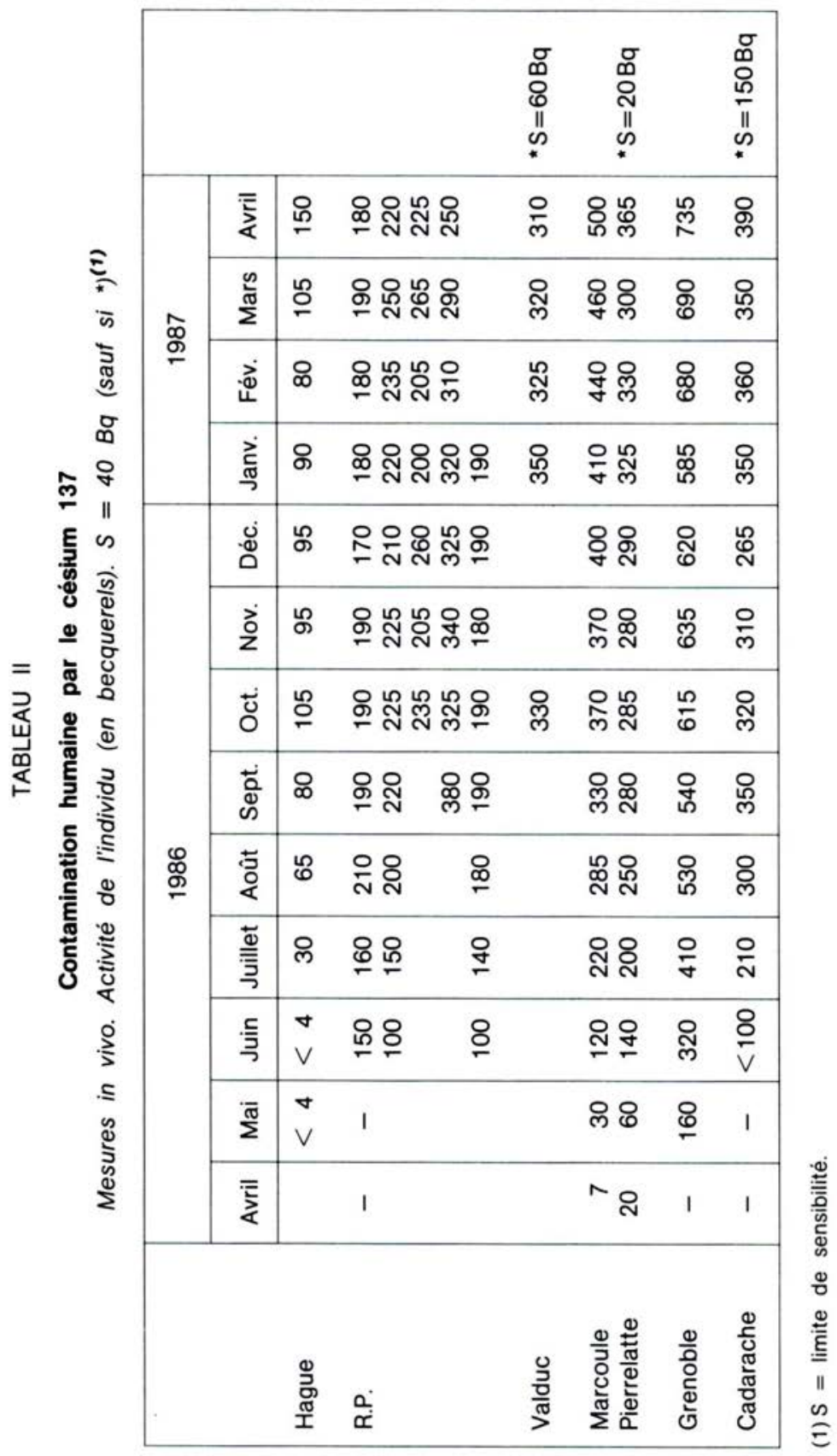


SUIVI DE LA CONTAMINATION HUMAINE EN CÉSIUM A LA SUITE DE L'ACCIDENT DE TCHERNOBYL

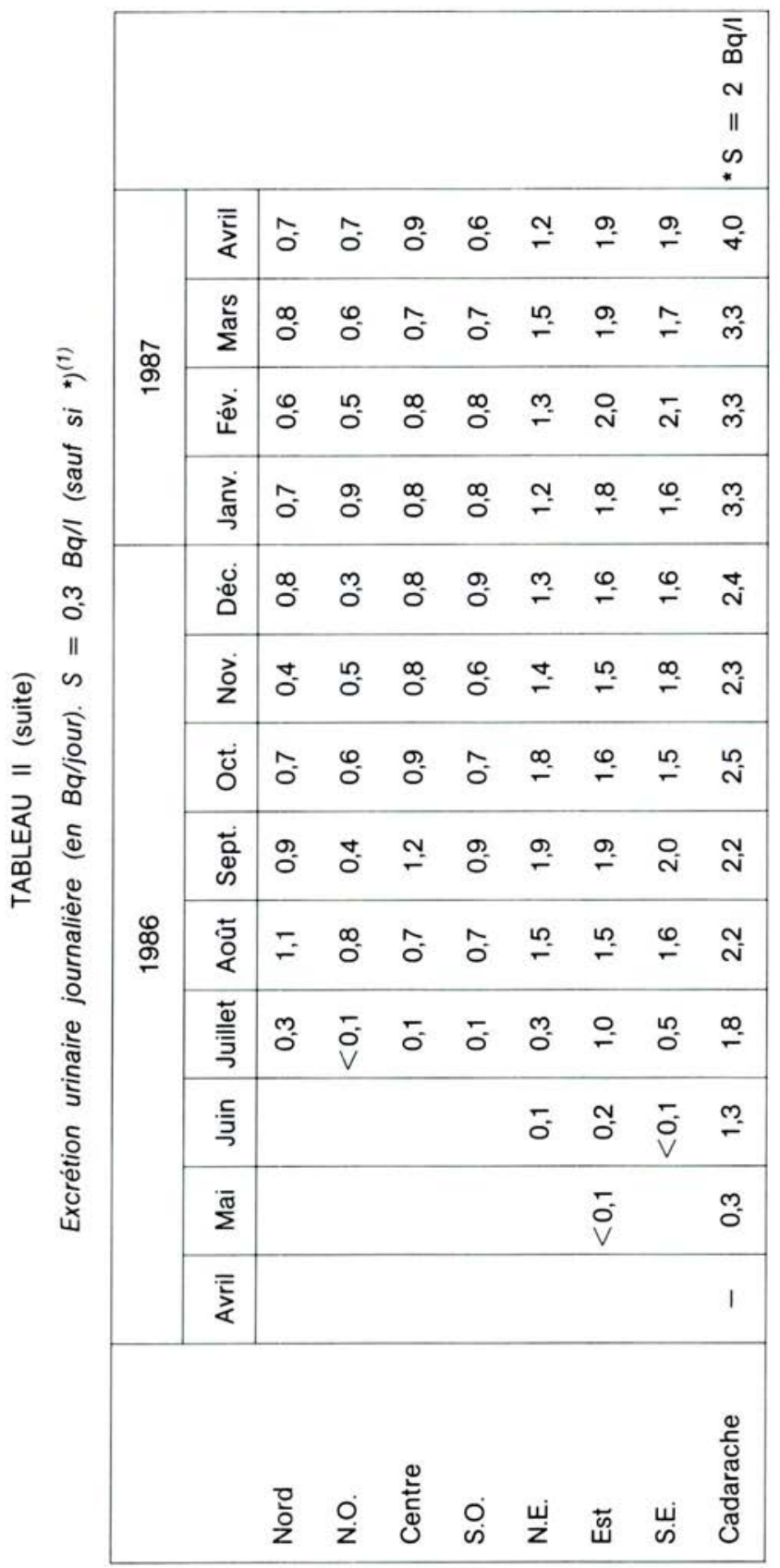

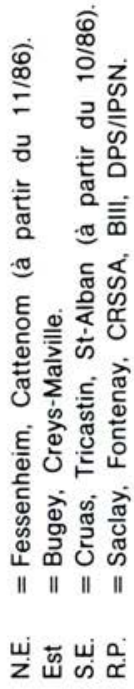

VOL. $22-\mathrm{N}^{\circ} 4$ 
SUIVI DE LA CONTAMINATION HUMAINE EN CESIUM A LA SUITE DE L'ACCIDENT DE TCHERNOBYL

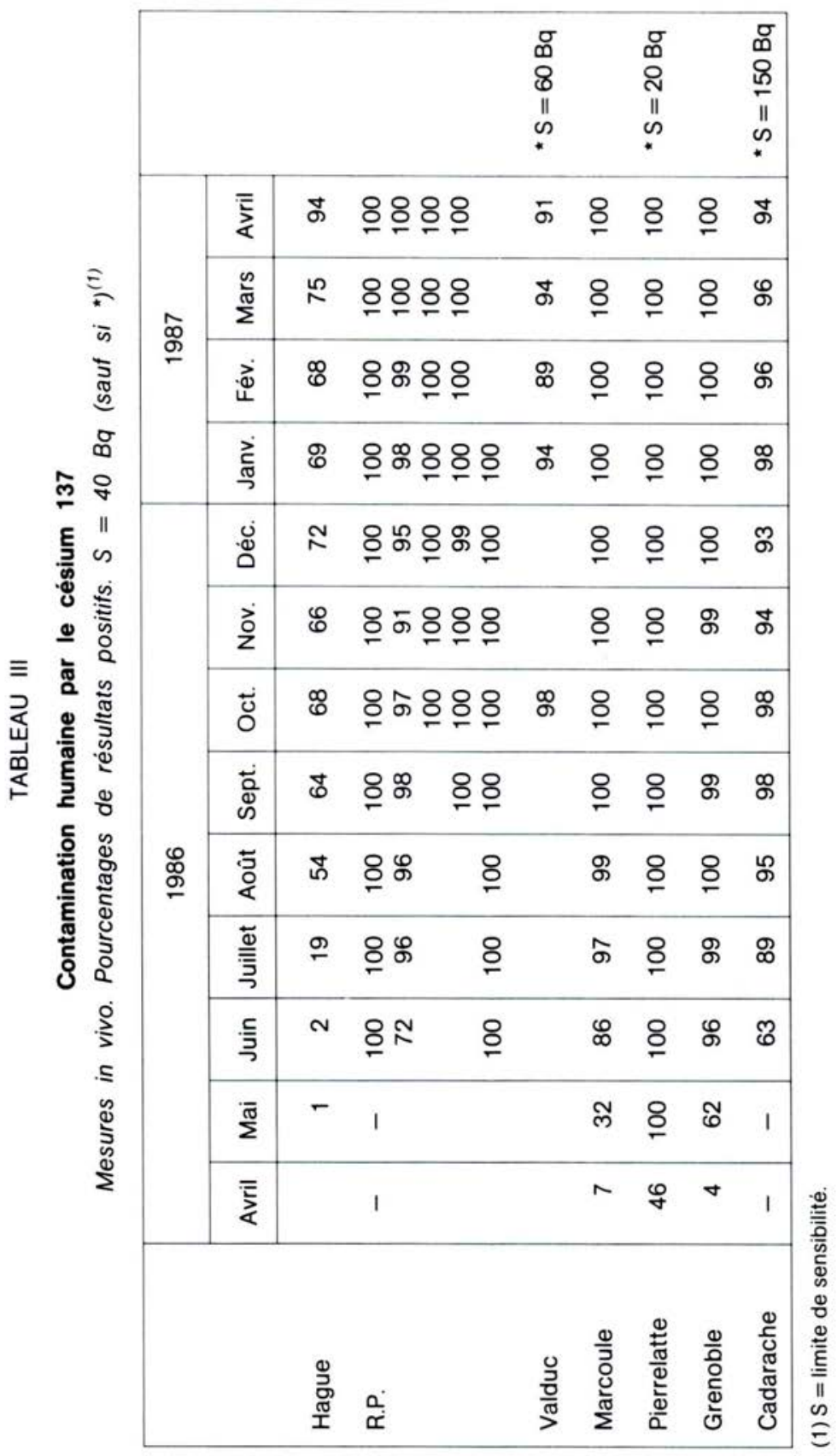


SUIVI DE LA CONTAMINATION HUMAINE EN CÉSIUM A LA SUITE DE L'ACCIDENT DE TCHERNOBYL

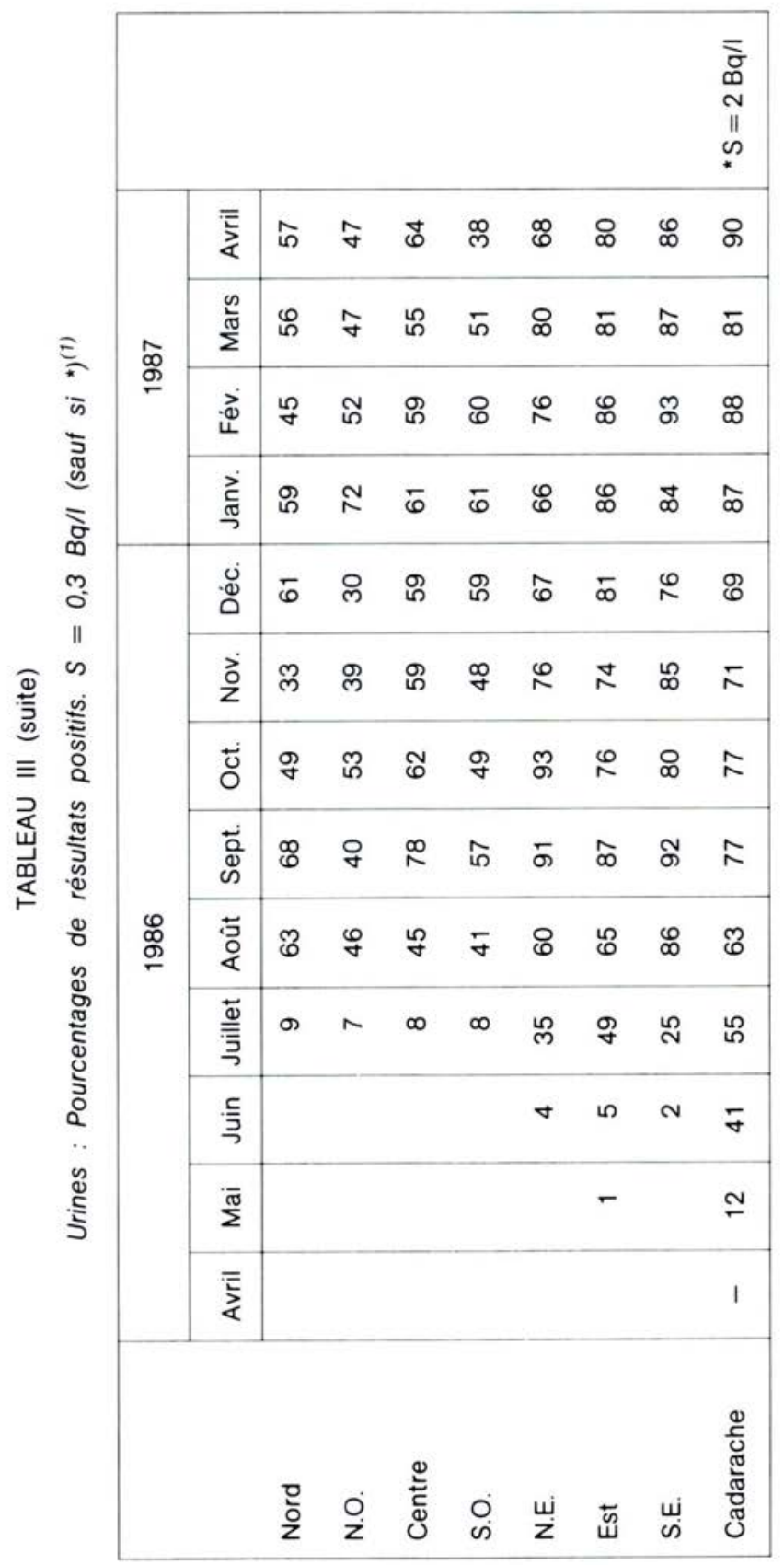

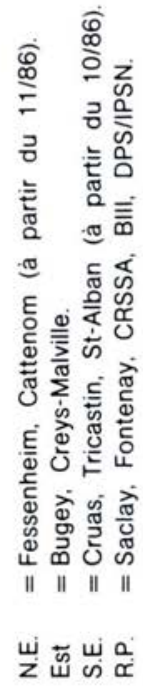

VOL. $22-\mathrm{N}^{\circ}{ }_{4}$ 
Fig. 2. - Mesures in vivo.
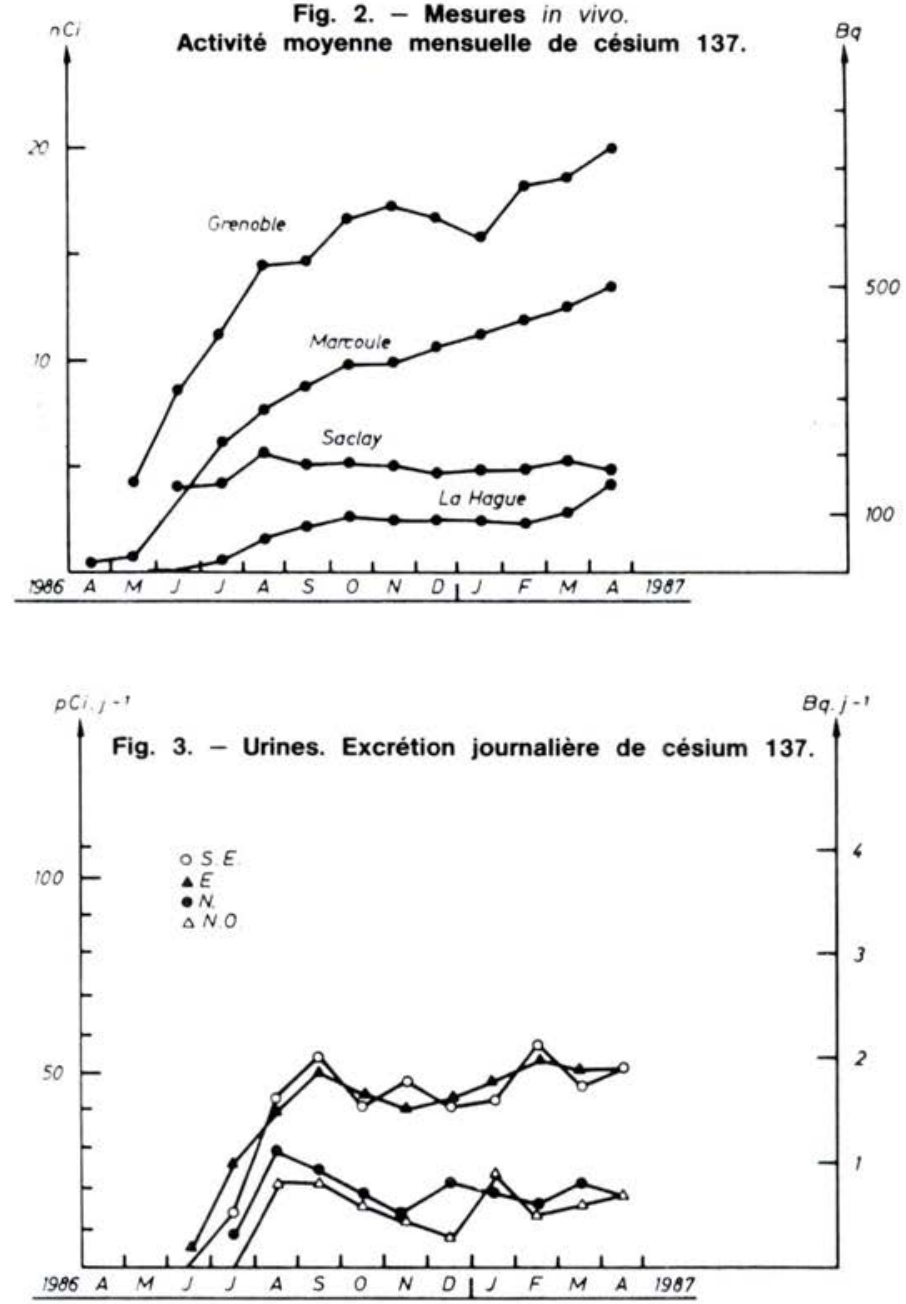

\section{Estimation de l'ingestion}

Le tableau IV et la figure 1 indiquent les activités de césium 137 absorbées en un an, calculèes à partir des résultats urinaires et in vivo. La comparaison des résultats montre un bon accord entre les deux méthodes utilisées, lorsqu'il s'agit de la même région. Aussi, pensons-nous que l'écart d'un facteur 2 à 6 entre les valeurs obtenues à l'est et à l'ouest du pays peut être considéré comme représentatif. 
TABLEAU IV

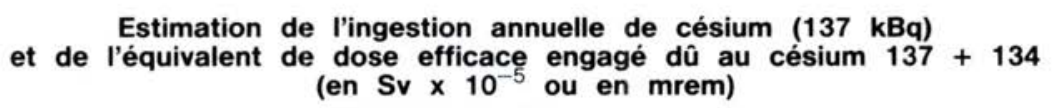

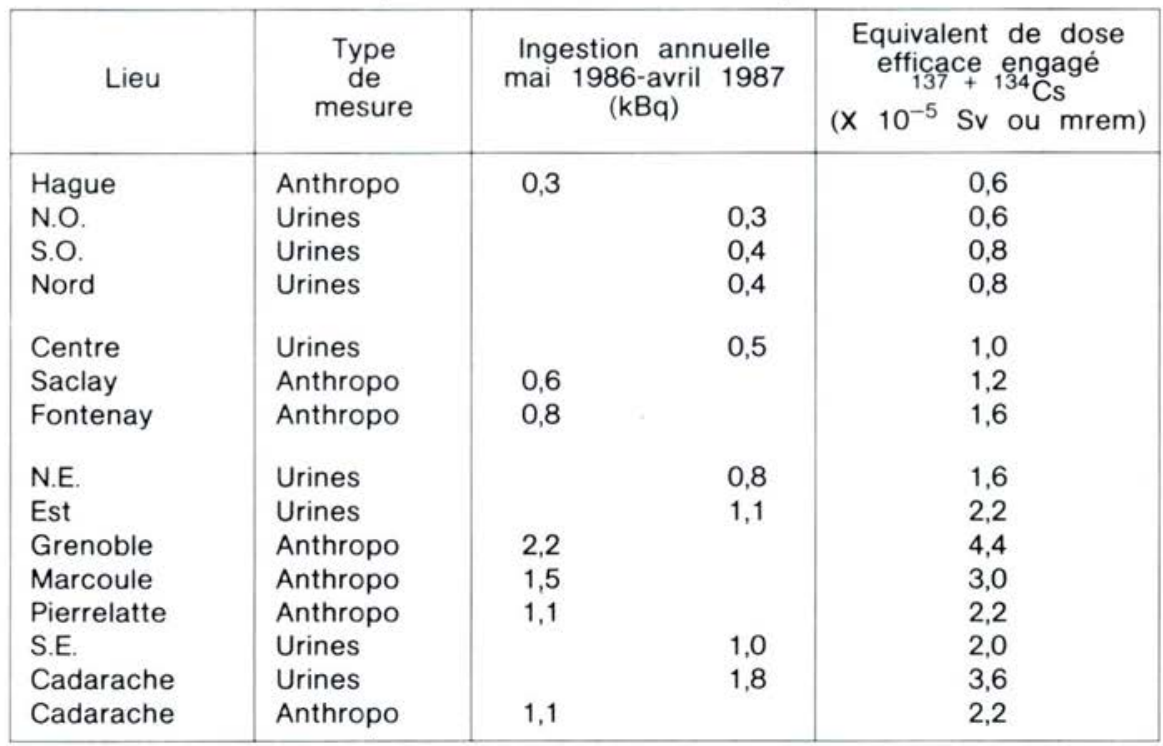

N.O. = Paluel, Flamanville.

S.O. = Le Blayais.

Nord $=$ Gravelines

Centre $=$ Dampierre, St-Laurent, Chinon.
N.E. = Fessenheim, Cattenom

(à partir de novembre 1986)

Est = Bugey, Creys-Malville.

S.E. = Cruas, Tricastin, St-Alban

(à partir d'octobre 1986)

Le tableau $V$ montre l'estimation de l'ingestion journalière moyenne de césium 137 au cours des différents mois. Les valeurs ne doivent pas être considérées individuellement, mais dans leur ensemble; en effet, le mode de calcul de l'ingestion moyenne mensuelle utilisant des différences de résultats de mesures eux-mêmes entachés d'une certaine erreur, tend à en amplifier les variations.

Les résultats montrent que l'incorporation a crû d'abord rapidement, puis s'est ralentie, mais il n'est pas certain que le maximum soit atteint partout après un an. II est intéressant de noter que les circuits de distribution alimentaire n'ont pas provoqué de diffusion rapide conduisant à une contamination uniforme de l'ensemble de la population. La contamination humaine est sans doute liée étroitement à celle du lait (fig. 4). Pour les laits les plus actifs, la contamination a nettement augmenté au cours de l'hiver ; ceci est probablement dû à la consommation de fourrage récolté au moment des retombées du printemps 1986 et donc plus actif. La contamination d'une ration alimentaire de la région parisienne évolue de la même façon. Dans le tableau $V$, 
SUIVI DE LA CONTAMINATION HUMAINE EN CESIUM A LA SUITE DE L'ACCIDENT DE TCHERNOBYL

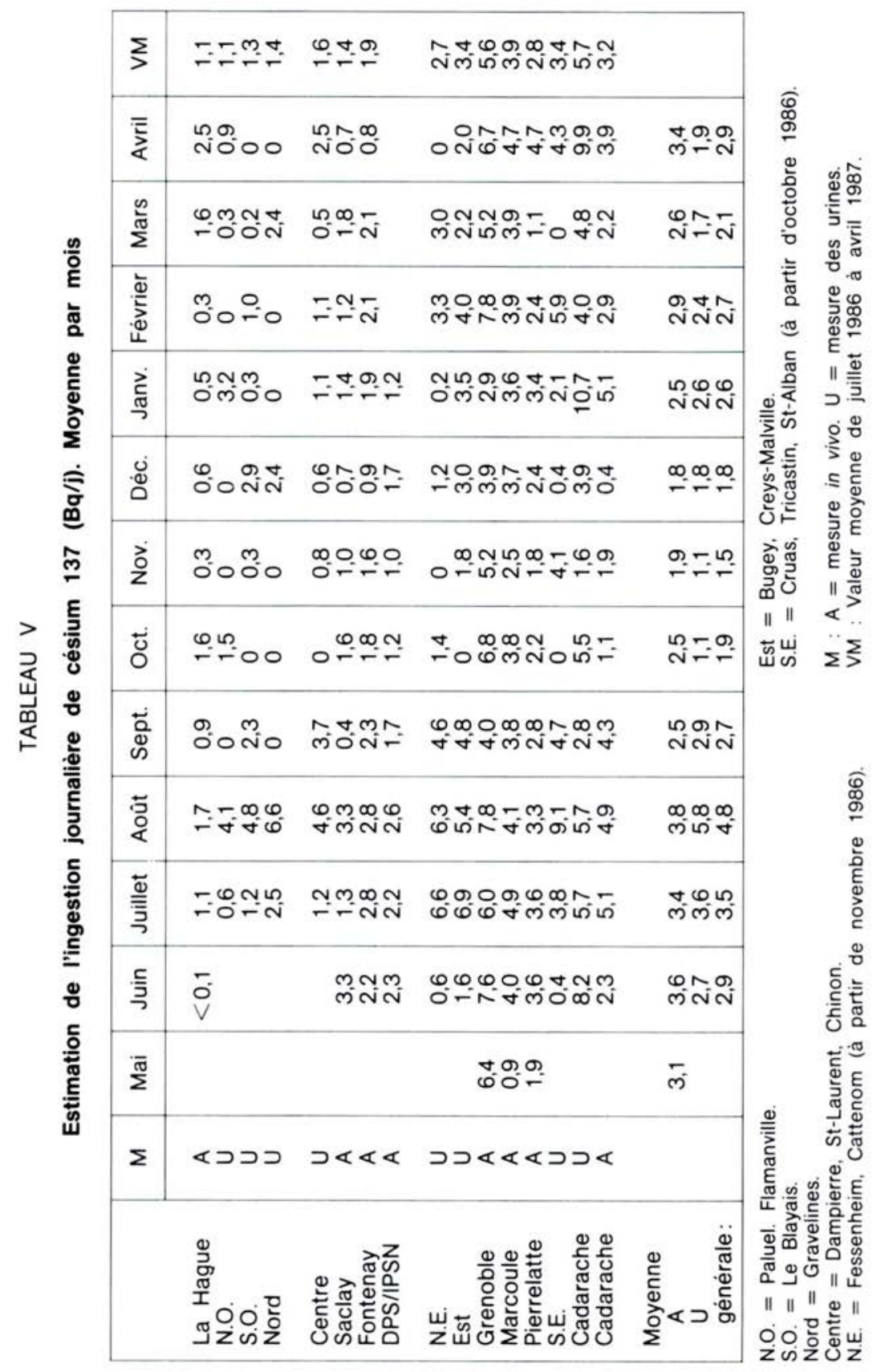


SUIVI DE LA CONTAMINATION HUMAINE EN CÉSIUM A LA SUITE DE L'ACCIDENT DE TCHERNOBYL $\rho C_{1} g^{-1}$

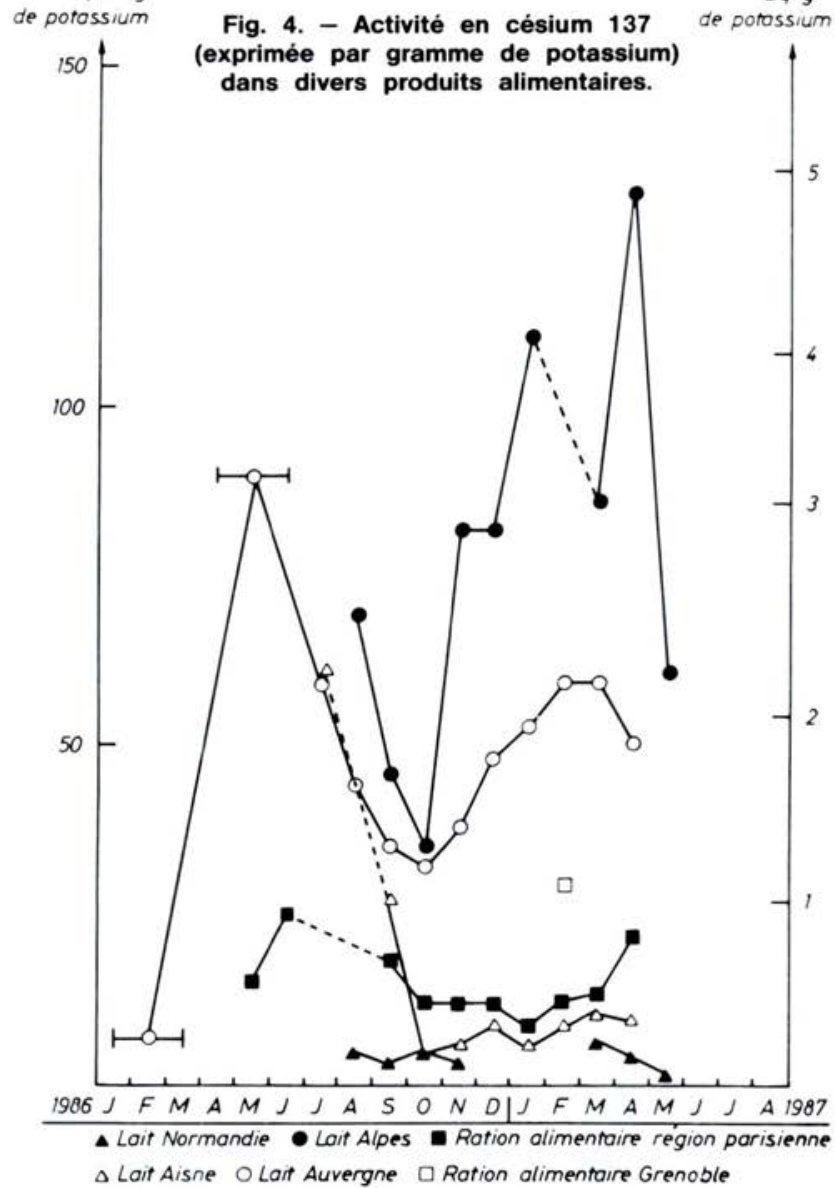

les résultats de la dernière ligne (moyenne générale) montrent que l'ingestion journalière est passée par un minimum vers la fin de l'année, évolution en accord avec les résultats de la figure 4 ; il s'agit sans doute d'un phénomène réel, car on le retrouve sur la figure 5 où sont reportées les valeurs de l'ingestion journalière calculées à partir des mesures in vivo et urinaires.

\section{Exposition}

Les résultats présentés concernent uniquement le césium 137. En réalité, il est accompagné d'un autre isotope, le césium 134. Le rapport 137/134 était d'environ 2 dans les retombées. En admettant que le rapport 137/134 soit resté constant et égal à 2 pendant l'année considérée, $1 \mathrm{kBq}$ de césium 137 ingéré délivre, avec le césium 134 qui l'accompagne, un équivalent de dose efficace engagé de $20 \mu \mathrm{Sv}$ ( $2 \mathrm{mrem})$. Les valeurs moyennes extrêmes (tableau IV) sont de $44 \mu \mathrm{Sv}(4,4 \mathrm{mrem})$ à Grenoble et $6 \mu \mathrm{Sv}(0,6 \mathrm{mrem})$ en 


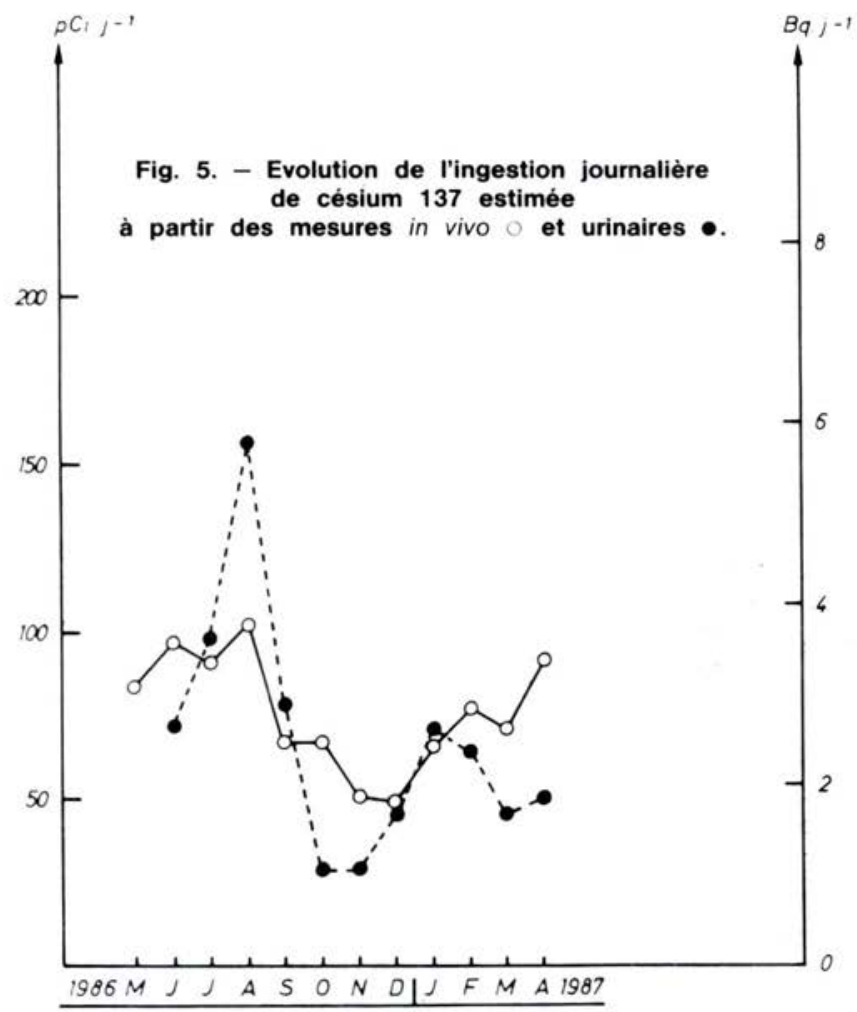

Normandie; ces valeurs peuvent être comparées à l'exposition moyenne annuelle de $170 \mu \mathrm{Sv}$ (17 mrem) due au potassium 40, élément naturel qui a la même répartition que le césium dans l'organisme.

En plus de ces valeurs moyennes, il est intéressant de connaître l'exposition des sujets les plus contaminés. Dans ce but, quelques détails supplémentaires concernant les mesures in vivo figurent au tableau VI.

- la colonne 3 indique que le rapport des activités maximales et moyennes varie de 1,6 à 10; en général, ce rapport est d'autant plus grand que l'activité moyenne est faible, et les valeurs maximales (col. 2) sont moins dispersées que les moyennes; ainsi les équivalents de doses efficaces engagés maximals sont voisins à La Hague, Saclay, Fontenay-aux-Roses, Pierrelatte et Cadarache;

- la colonne 6 indique l'estimation des équivalents de doses efficaces engagés pour les 5 incorporations maximales individuelles les plus élevées; compte tenu du fait que certaines personnes consomment les produits de leurs propres plantations ou pratiquent un "mode de vie écologique", ces valeurs exceptionnelles et leur nombre paraissent modérés. 
TABLEAU VI

Expositions maximales et exceptionnelles

\begin{tabular}{|c|c|c|c|c|c|c|c|}
\hline & 1 & 2 & 3 & 4 & 5 & 6 & 7 \\
\hline & $\begin{array}{c}\text { Activité } \\
\text { moyenne } \\
(\mathrm{kBq})\end{array}$ & $\begin{array}{c}\text { Activité } \\
\text { maximale } \\
(\mathrm{kBq})\end{array}$ & $\begin{array}{c}\text { Rapport } \\
2 / 1\end{array}$ & $\begin{array}{c}\text { Dose } \\
\text { eff. } \\
\text { moy. } \\
\left(10^{-5} \text { Sv }\right)\end{array}$ & $\begin{array}{c}\text { Dose } \\
\text { eff. } \\
\text { max. } \\
\left(10^{-5} \mathrm{~Sv}\right)\end{array}$ & $\begin{array}{c}\text { Dose } \\
\text { eff. } \\
\text { exc. } \\
\left(10^{-5} \text { Sv }\right)\end{array}$ & $\begin{array}{c}\text { Orgine de la } \\
\text { contamination } \\
\text { en col. } 6\end{array}$ \\
\hline La Hague & 0,9 & 9 & 10 & 0,6 & 6,0 & & \\
\hline Sacl & 2,0 & 8,4 & 4,2 & 1,2 & 5,0 & 19 & $\begin{array}{l}\text { Sujet turc. } \\
\text { Vacances en } \\
\text { Turquie en } \\
\text { juillet } 1986 \text {. }\end{array}$ \\
\hline Fontenay & 2,3 & 7,0 & 3 & 1,6 & 4,8 & - & \\
\hline B III & 2,6 & 8 & 3,1 & & & 19 & $?$ \\
\hline $\begin{array}{l}\text { DPS/IPSN } \\
\text { Marcoule }\end{array}$ & $\begin{array}{l}1,4 \\
3,9\end{array}$ & ${ }_{14}^{2,3}$ & $\begin{array}{l}1,6 \\
3,6\end{array}$ & 3,0 & 10,8 & 12 & \\
\hline Pierrelatte & 3,1 & 8,6 & 2,8 & 2,2 & 6,0 & 16 & $\begin{array}{l}\text { Consommation } \\
\text { de lait d'une } \\
\text { ferme }\end{array}$ \\
\hline Grenoble & 6,6 & 16,3 & 2,5 & 4,4 & 11,0 & 15 & $?$ \\
\hline Cadarache & 3,2 & 9,4 & 2,9 & 2,2 & 6,4 & & \\
\hline
\end{tabular}

Colonne 1: Somme des activités moyennes mensuelles, tirée du tableau II.

Colonne 2: Somme des activités maximales mensuelles.

Colonne 3: Rapport colonnes 2/1.

Colonne 4: Equivalent de dose efficace engagé moyen (cf. tabl. IV).

Colonne 5: Equivalent de dose efficace engagé maximal $=$ dose efficace moyenne (col. $4 \times$ rapport de la colonne 3).

Colonne 6: Equivalent de dose efficace engagé exceptionnel estimé à partir de points remarquables dans la liste des activités maximales d'un centre. Le calcul est effectué en faisant le rapport (valeur exceptionnelle / valeur moyenne du même mois), et en multipliant ce rapport par la dose efficace de la colonne 4.

A l'opposé de ces valeurs maximales, il est possible d'appliquer le même traitement aux valeurs minimales; il conduit à la conclusion que l'équivalent de dose efficace engagé minimal serait inférieur à $3 \mu \mathrm{Sv}(0,3 \mathrm{mrem})$ à La Hague et à $7 \mu$ Sv $(0,7$ mrem $)$ à Grenoble.

Le tableau VII montre la comparaison des estimations d'équivalents de doses efficaces engagés pour la première année après le passage du nuage; ces estimations sont obtenues par deux voies entièrement différentes: celles de cette étude reposent sur les mesures effectuées chez l'homme, les autres sont basées sur les mesures dans l'environnement [2, 3]. La colonne 2 est un bilan récapitulatif, publié dès le 27 juin 1986, des conséquences sanitaires des différentes voies d'atteinte pour l'adulte [2]. Ce bilan fait l'estimation pour tous les radionucléides et pour la première année, à partir des mesures effectuées pendant les premières semaines dans l'environnement: air, dépôt, chaîne alimentaire. La colonne 3, établie de la même façon, ne concerne que les césium 137 et 134 . Les résultats des colonnes 3 et 4 sont en bon accord et montrent que des méthodes d'approche très différentes sont utilisables pour estimer les conséquences d'un accident. Toutefois, l'objet de la radioprotection étant de connaître les conséquences sanitaires chez l'homme, les mesures obtenues chez celui-ci ont l'avantage 
de décrire ce qui se passe réellement, en confirmant ou en infirmant les résultats obtenus par des méthodes à but prévisionnel.

TABLEAU VII

Estimation des équivalents de dose efficace obtenus à partir de mesures dans l'environnement et chez l'homme (en Sv $\times 10^{-5}$ ou en mrem)

\begin{tabular}{|c|c|c|c|}
\hline$\stackrel{1}{\text { Région }}$ & $\stackrel{2}{\text { Environnement }}$ & $\begin{array}{c}3 \\
\text { Environnement }\end{array}$ & $\stackrel{4}{\text { Homme }}$ \\
\hline & $\begin{array}{c}\text { Bilan } \\
\text { global } \\
\text { au } 28.06 .86\end{array}$ & $\begin{array}{l}\text { Bilan partiel } \\
\text { au } 28.06 .86 \\
137+{ }^{134} \mathrm{Cs}\end{array}$ & $\begin{array}{c}\text { Bilan } \\
\text { du tableau IV } \\
{ }_{137}+134 \mathrm{Cs}\end{array}$ \\
\hline $\begin{array}{l}\text { Normandie } \\
\text { lle-de-France } \\
\text { Bourgogne } \\
\text { Grenoble } \\
\text { Cadarache }\end{array}$ & $\begin{array}{c}0,5 \\
2,5 \\
17 \\
2,5 \\
8\end{array}$ & $\begin{array}{l}0,2 \\
1 \\
0,5 \\
2 \\
2,5\end{array}$ & 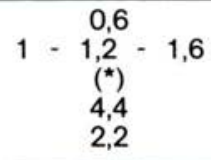 \\
\hline
\end{tabular}

Colonne 2: Bilan récapitulatif des conséquences sanitaires des différentes voies d'atteinte pour l'adulte estimé pour la première année après le passage du nuage, publié le 27 juin $1986[2,3]$.

Colonne 3: Bilan partiel ne concernant que les ${ }^{137}$ et ${ }^{134} \mathrm{Cs}$. Ces valeurs ont été utilisées pour établir le bilan global de la colonne 2 (F. LAYLAVOIX, communication personnelle).

Colonne 4 : Bilan obtenu dans cette étude (cf. tableau IV) après avoir effectué des mesures chez I'homme pendant une année.

(*) : Les calculs n'ont pas été effectués car certaines données manquent. Toutefois par comparaison des données du tableau II, l'équivalent de dose efficace peut être estimé à 2 ou $3.10^{-5}$ Sv ( 2 ou 3 mrem).

\section{CONCLUSION}

Les résultats de la surveillance systématique pratiquée sur le personnel travaillant dans les installations nucléaires peuvent être utilisés en cas de retombée imprévue pour obtenir des indications sur la contamination de la population, même lorsque cette contamination est très faible. Les résultats urinaires et in vivo donnent des informations du même ordre de grandeur. L'apparition et l'accroissement de cette contamination ont pu être mis en évidence grâce à l'extrême sensibilité des moyens de détection, et aussi parce que la contamination professionnelle en césium 137 et 134 de l'ensemble du personnel est négligeable.

\section{RÉFÉRENCES}

[1] INTERNATIONAL COMMISSION ON RADIOLOGICAL PROTECTION (ICRP). Limits for intakes of radionuclides by workers (ICRP Publication 30). Oxford: Pergamon Press, 1979, suppl. to part 1.

[2] LAYLAVOIX F., MADELMONT C., PARMENTIER N., ROBEAU D., WARTENBERG I. Estimation des conséquences sanitaires en France de l'accident nucléaire de Tchernobyl (26 avril 1986) à partir des mesures faites par le groupe CEA. Fontenay-aux-Roses: CEA, IPSN-DPS. Rapport DPS-86/03-SEAPS, 1986.

[3] Mesures de radioactivité dans l'environnement et évaluation de l'exposition des populations en France après l'accident de Tchernobyl. Radioprotection, 1987, 22 (1) 29-54.

[4] PIECHOWSKI J., MENOUX B. Rétention et excrétion des radionucléides après incorporation par inhalation chez l'homme adulte. Rapport CEA-R-5266, 1984, p. 105. 